\title{
Time Series Analysis of the Clinical Laboratory Test Result on Chemotherapy for Gastric Cancer
}

\author{
Noboru Sotoishi ${ }^{\mathrm{a}, \mathrm{c}}$, Takao Katsube ${ }^{\mathrm{b}}$, Kenji Ogawa ${ }^{\mathrm{b}}$, Shigeru Yakou ${ }^{\mathrm{a}}$, Kozo Takayama ${ }^{\mathrm{c}}$ \\ a Department of Pharmacy, ${ }^{\mathrm{b}}$ Department of Surgery, Tokyo Woman’s Medical University East Medical Center, Nishiogu \\ 2-1-10, Arakawa, Tokyo’ 116-8567, Japan; ' Department of Pharmaceutics, Hoshi University, Ebara 2-4-41, Shinagawa, \\ Tokyo 142-8501, Japan
}

Received, July 13, 2008; Revised, November 21, 2008; Accepted, December 24, 2008, Published, January $15,2009$.

\begin{abstract}
Purpose. Time series analysis may be helpful to estimate hematological data on gastric cancer patients who receive S-1, but untreated raw clinical data are not suitable for this approach. Hematological monitoring data interpolated by spline were analyzed by an attractor plot, which is a form of time series analysis. Methods. Hematological data of three gastric cancer patients were interpolated by cubic spline. The leave-one-out cross validation method was carried out and an attractor plot was adopted to evaluate red blood count (RBC) data. Results. Well-predicted data, such as RBC, changed slightly; however, data with great deviation, such as the white blood count (WBC), were poorly predicted. The reaction of marrow function to chemotherapy was observable by spline interpolation of RBC data. Furthermore, an attractor plot clarified the tendency of the interpolated hematological monitoring data. Conclusions. It is suggested that spline interpolation is effective as a pretreatment to analyze clinical data from a time series.
\end{abstract}

\section{INTRODUCTION}

Various clinical tests are conducted to evaluate the effectiveness and adverse reactions of most medications. For instance, when HMG-CoA-reducing enzyme inhibitors are prescribed to a patient, the amount of cholesterol in the blood is measured to elucidate the therapeutic activity. In addition, creatine kinase levels are monitored to prevent the onset of rhabdomyolysis. For the same reason, tumor markers, white blood cell count (WBC) and other tests are performed in cancer chemotherapy. Prescribers refer to clinical test results to decide whether to continue the medication. It is important to understand the tendency of clinical test results because they change over time. This tendency may be understood according to data plots; however, objective evaluation of this tendency is difficult because of the low frequency and irregular intervals of the test.

Recently, TS-1, containing tegafur, -chloro-2,4-dihydroxypyridine (CDHP) and potassium oxonate (Oxo), (S-1, Taiho Pharmaceutical Co. Ltd., Tokyo, Japan) has been used in chemotherapy for most gastric cancers. S- 1 is one such derivative that combines tegafur with two modulators of 5-fluorouracil (5-FU) metabolism. It is administrated twice daily for 28 days, followed by a 14-day washout period. Oral S-1 at the presently recommended doses produces a higher $\mathrm{C}_{\max }$ and a greater AUC of plasma 5-FU than continuous protracted intravenous infusion of 5-FU (1). When S-1 is prescribed to a patient, in order to prevent untoward action, liver, renal and marrow function should be checked. In particular, hematological monitoring is very important because myelosuppression, mainly leucopenia, is a dose-limiting toxicity (DLT) of S-1. The overall incidence of adverse reactions in phase II studies was $74.3 \%$, and that of grade 3 or worse was $14.9 \%$. The main adverse reactions were myelosuppression and gastrointestinal toxicities; therefore, careful hematological monitoring is necessary. The dosage of $\mathrm{S}-1$ is adjusted for marrow function $(2,3)$. It is considered that objective estimation of hematological monitoring makes it possible to regulate the S-1 dose; however, it is difficult to extract important information from the data. To solve this problem, time series analysis may be helpful. Time series analysis is used to forecast future events based on known past events; however, untreated raw clinical data are not suitable for this approach.

Corresponding Author: Noboru Sotoishi, Department of Pharmacy, Tokyo Woman's Medical University East Medical Center, Nishiogu 2-1-10, Arakawa, Tokyo 116-8567, Japan e-mail: sotoph@dnh.twmu.ac.jp 
Spline is a piecewise polynomial function that is connected smoothly and has several continuous derivatives. Additionally, it passes exactly through each vertebral point (i.e., it has zero errors locally around the chosen points) (4).

This method has been introduced in various fields, such as creating sea floor topography from satellite data (5). In the medical field, it is beginning to be used in areas such as basic research $(6,7)$, diagnostic imaging (8).

The aim of this study was to investigate the possibility of spline interpolation. Hematological monitoring data were interpolated by cubic spline and the interpolated data were estimated from their correlation with actual data by way of a leave-one-out cross validation (LOOCV). Furthermore, an attractor plot was applied as time series analysis in order to clarify the tendency of the interpolated hematological monitoring data.

\section{METHODS}

The hematological data of three patients who had received S-1 administration over 2 years period were investigated. White blood cell count (WBC), red blood cell count (RBC), hemoglobin ( $\mathrm{Hb}$ ), hematocrit (Ht), mean corpuscular volume (MCV), mean corpuscular hemoglobin $(\mathrm{MCH})$, mean corpuscular hemoglobin concentration (MCHC), platelet (PLT), coefficient of variation of the red blood cell distribution width (RDW-CV), platelet distribution width (PDW), and mean platelet volume (MPV) were interpolated by cubic spline. A data NESIA (Yamatake Corp., Tokyo, Japan), computer software for experimental data analysis, was used for spline interpolation. Hematological data of three patients was interpolated. In each examination, a prediction was made every seven days. The background of the patients is shown below. This study was approved by the ethics committee of Tokyo Woman's Medical University.

\section{Case A}

Birth date: May 13, 1939; Sex: Male; Height: 164cm; Weight: 68.3kg; BSA: $1.70 \mathrm{~m}^{2}$; Disease staging: T2, N3, H1, P0, M1; Pathology: Poorly diff. adenocarcinoma; Observation time: September 17, 2003 to October 13, 2006.

\section{Case B}

Birth date: March 4, 1941; Sex: Male; Height: 175cm; Weight: 60.0kg; BSA1.68m²; Disease staging: T2, N2, H1, P0; Pathology: Poorly diff. adenocarcinoma; Observation time: July 24, 2004 to October 27, 2006.

\section{Case C}

Birth date: September 26, 1939; Sex: Male; Height: 159cm; Weight: 60.9kg; BSA $1.59 \mathrm{~m}^{2}$; Disease staging: After gastric resection T2, N2, H0, P0, M0, Cy0; Pathology: Papillary adenocarcinoma; Observation time: January 9, 2004 to October 20, 2006.

The leave-one-out cross-validation method was carried out using software preinstalled in data NESIA. An attractor plot was made to evaluate the tendency implicit in the RBC data in detail. The attractor plot is constructed by joining points defined by the time between a set of data $\left(\mathrm{X}_{\mathrm{t}}, \mathrm{X}_{\mathrm{t}}\right.$ $\left.{ }_{+1}\right)$ and subsequent data $\left(X_{t+1}, X_{t+2}\right)$.

\section{RESULTS}

\section{Predictive accuracy of spline interpolation}

Typical examples for the data measured and predicted by spline interpolation in case $\mathrm{A}$ are shown in figure 1 . The LOOCV plot for spline interpolation in case A is shown in figure 2. The correlation coefficients (r) for RBC, $\mathrm{Hb}, \mathrm{MCV}$, and RDW-CV were high $(>0.89)$ although the prediction of WBC, MCHC, PLT, and PDW was poor. $(r=0.4-0.6)$ The well-predicted data was changed slightly; however, data with great deviation were poorly predicted. Typical examples for the predicted outcomes and LOOCV of patients $\mathrm{B}$ and $\mathrm{C}$ are shown in figures 3, 4, 5 and 6 , respectively. The results observed for patients $B$ and $\mathrm{C}$ were almost identical to those of patient $\mathrm{A}$.

\section{Relation between chemotherapy and RBC}

As the RBC data were predicted quantitatively, the effect of chemotherapy on RBC data was investigated, considering clinical events.

Figure 7 shows three prescriptions of chemotherapy applied for case A. [i] is combined therapy of $\mathrm{S}-1 \quad$ : $70.8 \mathrm{mg} / \mathrm{m}^{2} \quad$ 4-week medication/2-week rest and cisplatin : $58.8 \mathrm{mg} / \mathrm{m}^{2}$ on day 8. [ii] is combined therapy of $\mathrm{S}-1$ : $70.4 \mathrm{mg} / \mathrm{m}^{2}$ 2-week medication/1-week rest and CPT- $11: 70.6 \mathrm{mg} / \mathrm{m}^{2}$ on day 1 and 8 , and [iii] is combined therapy of $\mathrm{S}-1: 58.7 \mathrm{mg} / \mathrm{m}^{2}$ 2-week medication/1-week rest and CPT- $11: 58.8 \mathrm{mg} / \mathrm{m}^{2}$ on day 1 and 8. Changes of prescription from [i] to [ii] and [ii] to [iii] were implemented because of untoward actions, such as eczema. Although the RBC gradually decreased in [i] and [ii], it recovered in [iii].

In case $\mathrm{B}$, the chemotherapy prescription was changed repeatedly, as shown in figure 8. Although S-1 and cisplatin were administrated together from 
the initial day to the day 268, the tumor progressed, and then chemotherapy was changed to the combined use of irinotecan and cisplatin. Furthermore, metastasis was checked and therapy was changed to paclitaxel single therapy. RBC values decreased gradually until day 541, except for a short period of irinotecan and cisplatin treatment; however, after changing to paclitaxel single therapy, RBC began to increase, and on day 700 , it had recovered to the value before beginning chemotherapy.

In case $\mathrm{C}$, administration of S-1 was restricted by untoward action. $50.3 \mathrm{mg} / \mathrm{m}^{2}$ of $\mathrm{S}-1$ was administered to this patient every other day, but this administration schedule was not effective in view of the pharmacokinetics. Figure 10 also shows that S-1 administration did not affect RBC; however, when the prescription was changed to the combined use of S-1 (administration for three weeks followed by one-week washout) and cisplatin or irinotecan, the RBC value decreased.

In these three cases, the reaction of marrow function to chemotherapy was observable by spline interpolation of RBC data.

\section{Evaluation of RBC data by attractor plot}

The actual measurement data were converted into data which are easy to analyze by time series;
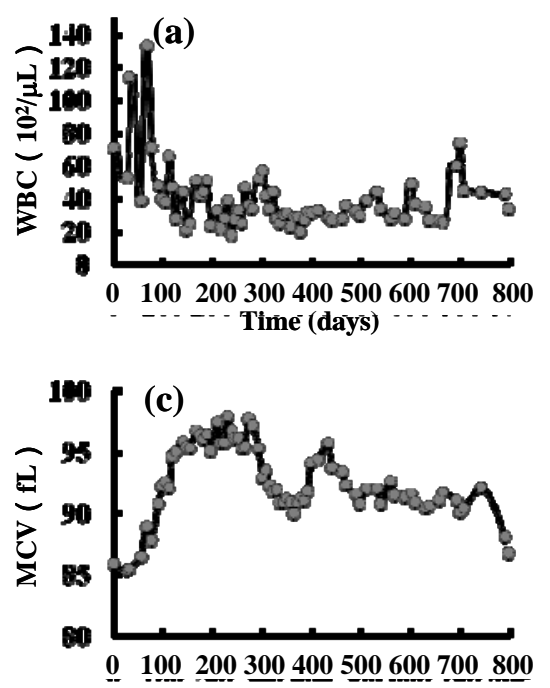

therefore, various analysis methods have become useful. An attractor plot was adopted to evaluate RBC data. Figures 10-12 are attractor plots of RBC data in case $\mathrm{A}, \mathrm{B}$, and $\mathrm{C}$. It was necessary to balance the data to equalize the observation intervals using this method. Data interpolated by spline were used for this qualification. There is no time axis in this graph as these plot points shift over time. The arrows in the figures are starting points of the attractor plot.

In case $\mathrm{A}$, the points shifted to the lower left from the upper right in [i]. In [ii], the plot was concentrated in the lower left. In [iii], it shifted to the center from the lower left. In other words, a remarkable reduction of RBC was shown in [i]. It remained low at $300-350 \cdot 10^{4} / \mathrm{mm}^{3}$ in [ii] and recovered in [iii]. The attractor plot therefore clarified the tendency of RBC data. Moreover, it indicated that different chemotherapy changed the attractor.

In case $\mathrm{B}$, because the administration period was short, the respective chemotherapy could not be analyzed, but the attractor plot implied that the plot shifted corresponding to the intensity of chemotherapy. When it was divided into a 300-day period, the tendency of a rise or descent was clear.
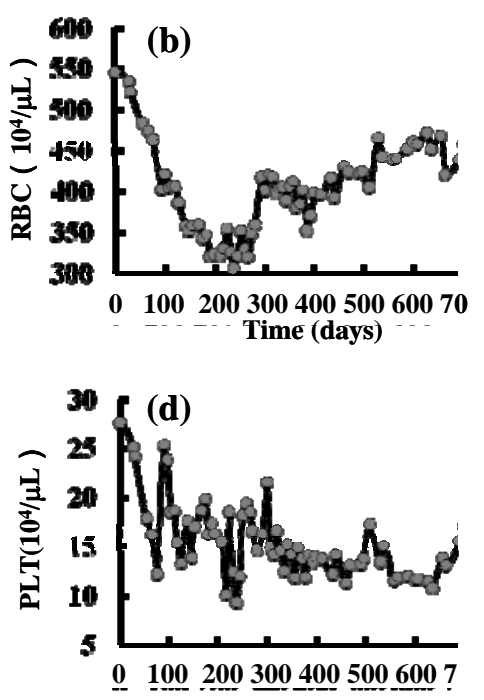

Figure 1. Time course (days) of actual and predicted hematological data in case A. Representative data was presented in this figure. Keys: Symbols and line represent observed and predicted values, respectively; (a) WBC, (b) RBC, (c) MCV, (d) PLT. 
In case $\mathrm{C}$, when $\mathrm{S}-1$ was prescribed every other day, the plot shifted in the range from 280 to 350, but after changing to strong chemotherapy, the plot shifted to the lower left rapidly.
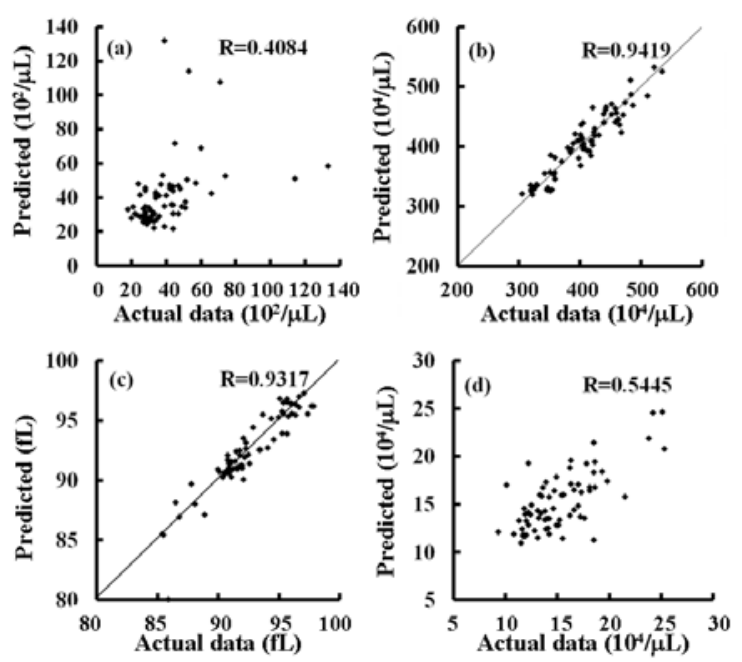

Figure 2. Leave-one-out cross-validation plots of hematological data measured and predicted by cubic spline in case A. (a) WBC, (b) RBC, (c) MCV, (d) PLT.

\section{DISCUSSION}

The interpolation method by spline was examined. Cubic spline was the best approach of various interpolation methods in this study. The interval of prediction was every seven days. The accuracy of prediction falls with an extremely shorter interval than the actual measurement; therefore, the prediction interval was determined in consideration of the life period of blood cells.

The dispersed clinical examination data were set to periodical data by cubic spline; therefore, the following can be expected.

1. It is easy to identify the tendency of clinical data.

2. Relevance between medical treatment and clinical data can be clarified.

3. Past clinical data can be generalized and their relation with other cases can be discussed.

4. Various analysis approaches are available even if there are few samples.

Some items on the blood test was well-predicted and poorly predicted by cubic spline. Data with great deviation, such as WBC, had an unclear tendency. The large deviations observed in
WBC in figures 1,3 and 5 are considered the influence of steroids that were administered with chemotherapeutic drugs; consequently, WBC was poorly predicted. Data that changed slightly, such as RBC, were well-predicted. This kind of data is suitable for evaluating tendency in clinical tests. In figures 7, 8 and 9, interpolated RBC data were concerned with clinical events. Generally, marrow function is evaluated by the reticulocyte count; however, the results of this study showed that acquiring detail information from RBC data can also estimate marrow function.
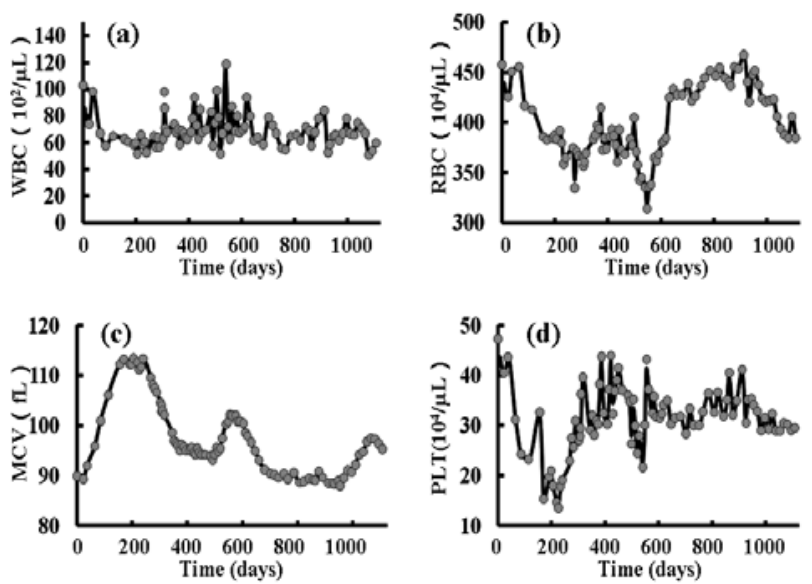

Figure 3. Time course (days) of actual and predicted hematological data in case B. Representative data was presented in this figure. Keys: For abbreviations see Figure 1.

Recently, pharmacokinetic pharmacodynamic (PK/ PD) models for the hematological toxicity of chemotherapy have been reported; absolute neutrophils counts (ANCs) are predicted using those models. This is an approach to construct a linear model for the time course of nadir neutrophils from numerous case data (9-12). Since our approach differs in not using the linear model, it is suitable for analysis of data measured in individual patients. Moreover, the nadir value of the neutrophils count is generally not measured in clinical treatment.

Various hematological data were interpolated by cubic spline. $\mathrm{Hb}$ and $\mathrm{Ht}$ are data related to $\mathrm{RBC}$ and have similar data profiles. $\mathrm{Hb}$ data are clinically used as indicators of anemia; however, it 
is considered that RBC should be investigated when evaluating myelosuppression. Other data (MCH etc.) should also be used to attempt time series analysis. It is important for many interpolated data to be analyzed by time series to identify how to analyze them.
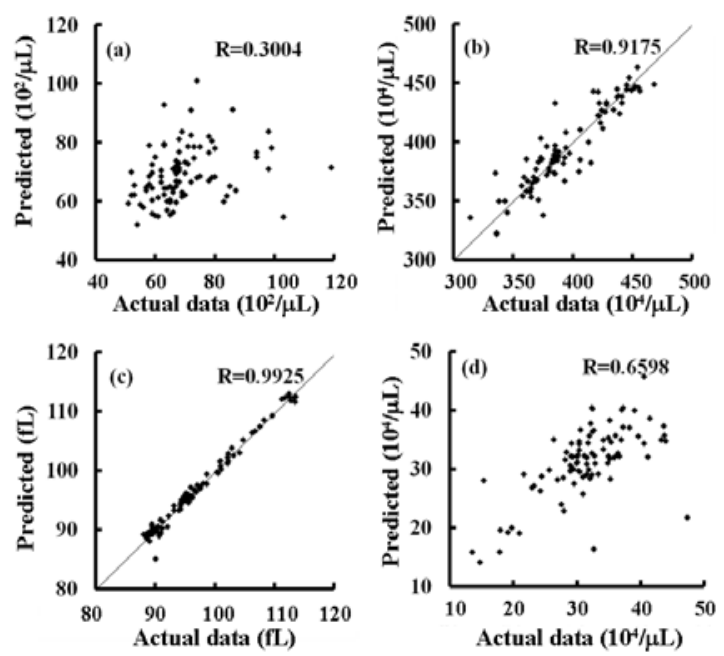

Figure 4. Leave-one-out cross-validation plots of hematological data measured and predicted by cubic spline in case B. (a) WBC, (b) RBC, (c) MCV, (d) PLT.

Interpolated RBC data were analyzed by attractor plot (see figures 10-12). The attractor plot shifted in the direction of the lower left when strong chemotherapeutic drugs were administered. In contrast, it shifted to the upper right with weaker drugs. Thus, the attractor plot corresponded to the clinical event. Although it was combination therapy, RBC decreased when administering high-dose S-1 in case A. The dosage of S-1 at the first prescription is distributed in only three steps $(40 \mathrm{mg}$, $50 \mathrm{mg}$ and $60 \mathrm{mg} /$ time) with the body surface area, but it is considered necessary to adjust the dose more minutely. Because the blood level of 5-FU in S-1 administration is very high. It was reported that 2-week administration followed by 1-week rest seemed more tolerable and safer than 4-week administration followed by a 2-week rest $(13,14)$. Furthermore, S-1 was prescribed for patients at a dose of $35-40 \mathrm{mg} / \mathrm{m}^{2}$ in a European trial (15); therefore, the usage of S-1 should be selected carefully and many factors considered to adjust the dose. It is thought that interpolated RBC is one of the factors that can be considered by attractor plot. An attractor plot is not the final step in time series analysis and other approaches should be considered; however, it was able to confirm that spline interpolation was useful as a pretreatment of time series analysis.
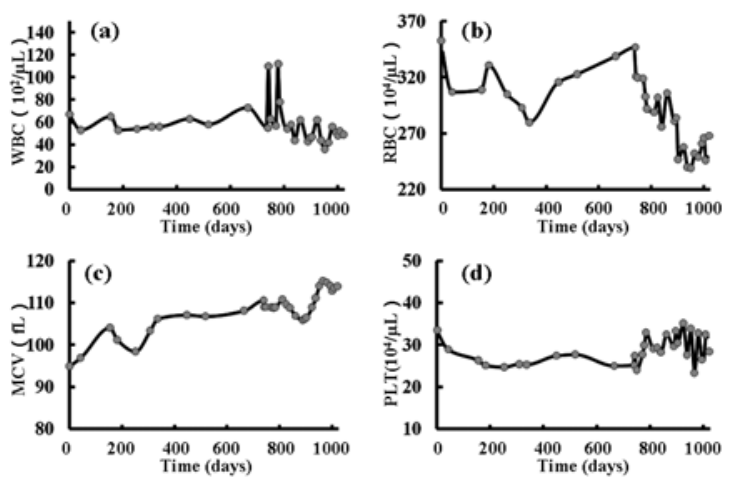

Figure 5. Time course (days) of actual and predicted hematological data in case $\mathrm{C}$. Representative data was presented in this figure. Keys: For abbreviations see Figure 1.
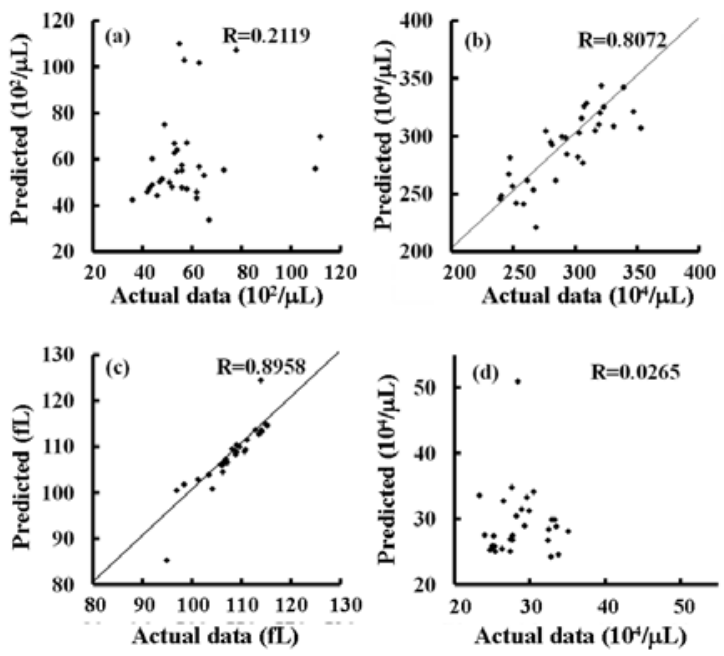

Figure 6. Leave-one-out cross-validation plots of hematological data measured and predicted by cubic spline in case C. (a) WBC, (b) RBC, (c) MCV, (d) PLT.

In this study, clinical hematological data were interpolated by cubic spline and an attractor plot was attempted to analyze the tendency of RBC. It is suggested that spline interpolation is effective as a pretreatment before analyzing clinical data by time series. To predict untoward reactions to chemotherapy, other factors in hematological data should be studied using various analysis methods. 


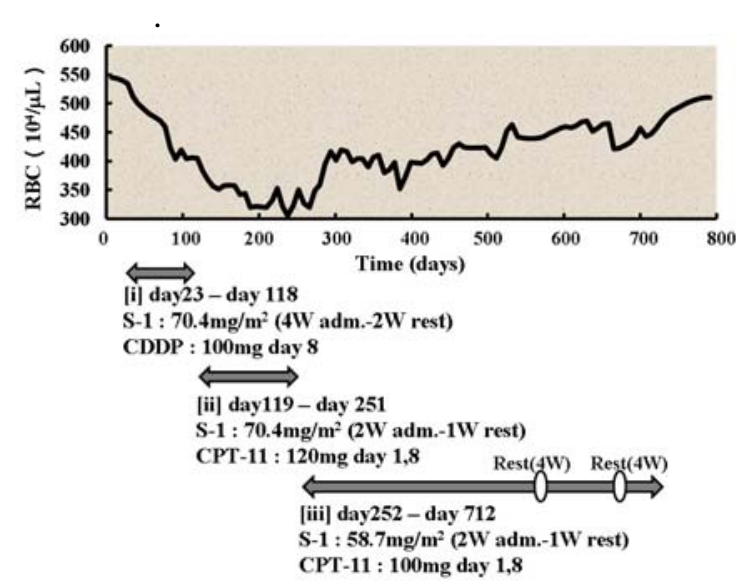

Figure 7. The relation of predicted RBC data and chemotherapy in case A

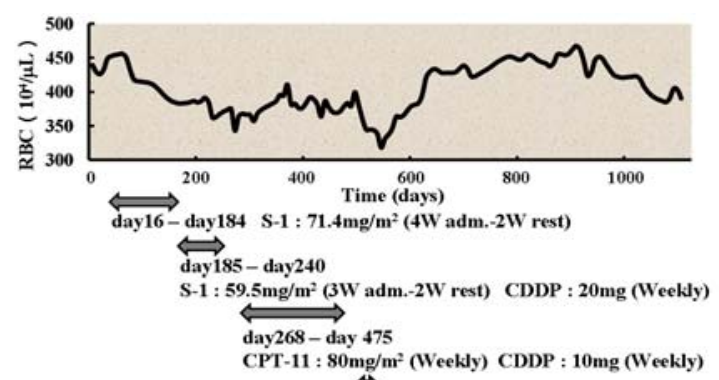

day 485 - day 541 S-1 : $71.4 \mathrm{mg} / \mathrm{m}^{2}$ (2W adm.-1W rest) CPT-11 : 80mg (Biweekly)

$$
\stackrel{\text { day542-day926 TXL: }}{\underset{1}{60 \rightarrow 80 \rightarrow 150 \mathrm{mg}}}
$$$$
\text { day947 - S-1 : 59.5mg/m2(2W adm. 1W rest) TXL : 120mg (Biweekly) }
$$

Figure 8. The relation of predicted RBC data and chemotherapy in case B

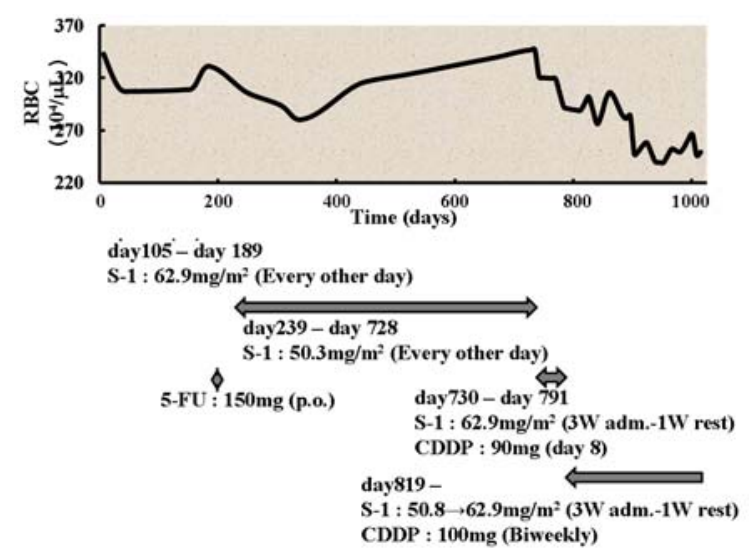

Figure 9. The relation of predicted RBC data and chemotherapy in case $\mathrm{C}$

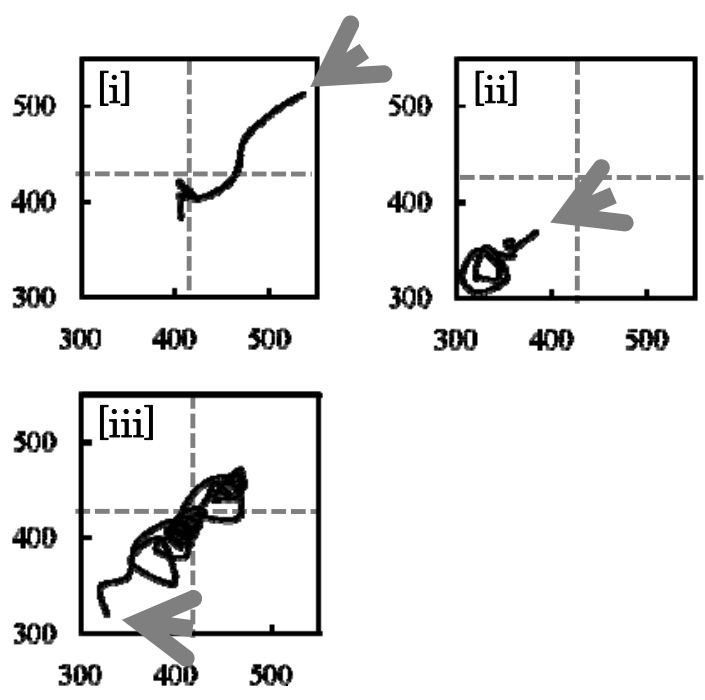

Figure 10. Attractor plots of $\mathrm{RBC}$ data predicted by cubic spline in case $\mathrm{A}$. The data $\mathrm{RBC}_{(\mathrm{t})}$ are plotted in horizontal axis, and the data $\mathrm{RBC}_{(\mathrm{t}+1)}$ are plotted in vertical axis. The arrows in this figure indicate starting points of attractor plots. [i], S-1: $70.4 \mathrm{mg} / \mathrm{m}^{2}$ four weeks administration and subsequently two weeks rest. CDDP: $100 \mathrm{mg}$ on day 8 . [ii], S-1: $70.4 \mathrm{mg} / \mathrm{m}^{2}$ two weeks administration and subsequently one week rest. CPT-11: $120 \mathrm{mg}$ on day 1 and 8 . [iii], S-1: $58.7 \mathrm{mg} / \mathrm{m}^{2}$ two weeks administration and subsequently one week rest. CPT-11: $120 \mathrm{mg}$ on day 1 and 8.
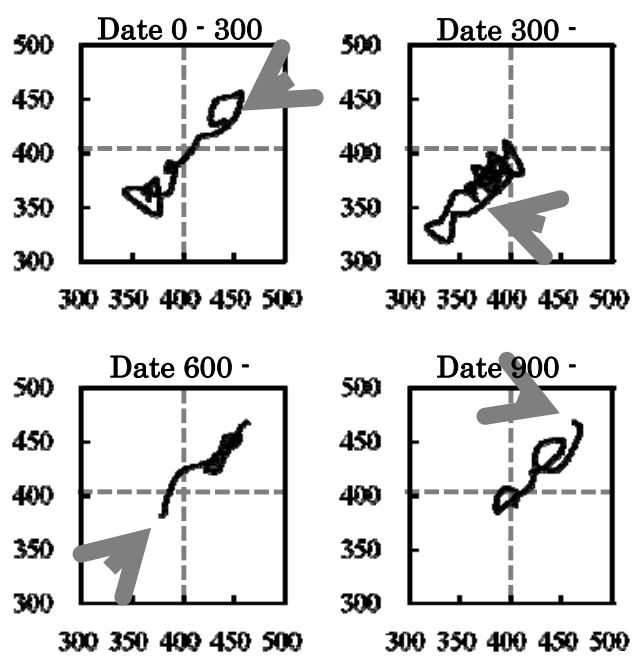

Figure 11. Attractor plots of RBC data predicted by cubic spline in case $\mathrm{B}$. The data $\mathrm{RBC}_{(\mathrm{t})}$ are plotted in horizontal axis, and the data $\mathrm{RBC}_{(\mathrm{t}+1)}$ are plotted in vertical axis. The arrows in this figure indicate starting points of attractor plots. 

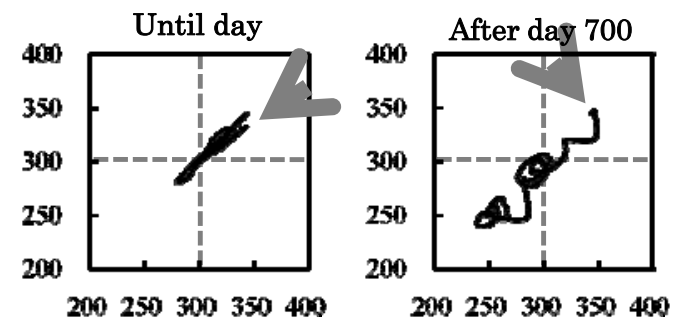

Figure 12. Attractor plots of RBC data predicted by cubic spline in case $\mathrm{C}$. The data $\mathrm{RBC}_{(\mathrm{t})}$ are plotted in horizontal axis, and the data $\mathrm{RBC}_{(\mathrm{t}+1)}$ are plotted in vertical axis. The arrows in this figure indicate starting points of attractor plots.

\section{REFERENCES}

[1] Yamada, Y., Hamaguchi, T., Goto, M., Muro, K., Matsumura, Y., Shimada, Y., Shirao, K. and Nagayama, S. Plasma concentrations of 5-fluorouracil and $\mathrm{F}$ - $\beta$-alanine following oral administration of $\mathrm{S}-1$, a dihydropyrimidine dehydrogenase inhibitory fluoropyrimidine, as compared with protracted venous infusion of 5-fluorouracil. Br J Cancer, 89: 816-820, 2003.

[2] Taguchi, T., Inuyama, Y., Kanamaru, R., Hasegawa, K., Aiazawa, S., Niitani, H., et al. Phase I study of S-1. Jpn J Cancer Chemother, 24: 2253-2264, 1997.

[3] Maehara, Y. S-1 in gastric cancer: a comprehensive review. Gastric Cancer, 6(Suppl 1): 2-8, 2003.

[4] De Boor, C. A Practical Guide to Spline. Springer Verlag, New York, 2001.

[5] Sandwell, D. T. Biharmonic spline interpolation of GEOS-3 and SEASAT altimeter data. Geophysical Research Letters, 14(2): 139-142, 1987.

[6] Takayama, K. Multivariate spline interpolation as a novel method to optimize pharmaceutical formulations. Pharmazie, 59( 5): 392-395, 2004.

[7] Arai, H., Suzuki, T., Kaseda, C., Ohyama, K. and Takayama, K. Bootstrap re-sampling technique to evaluate the optimal formulation of theophylline tablets predicted by non-linear response surface method incorporating multivariate spline interpolation. Chem. Pharm. Bull., 55(4): 586-593, 2007.

[8] Benson, P. Yang, Carina, W. Yang and Stephen, L. Ondra A novel mathematical model of the sagittal spine. Spine, 32(4): 466-470, 2007.

[9] Hing, J., Perez-Ruixo, J.J., Stuyckens, K., Soto-Matos, A., Lopez-Lazaro, L. and Zannikos, P. Mechanism-based pharmacokinetic/ pharmacodynamic meta-analysis of Trabectedin ( ET-743, Yondelis ) induced neutropenia. Clin. Pharmacol. Ther., 83(1): 130-143, 2008.

[10] van Kesteren, C., Zandvliet, A.S., Karlsson, M.O., Mathot, Ron, A.A., et al. Semi-physiological model describing the hematological toxicity of the anti-cancer agent indisulam. Investigational New Drugs, 23: 225-234, 2005.

[11] Sandstrom, M., Lindman, H., Nygren, P., Johansson, M., Bergh, J. and Karlsson, M.O. Population analysis of the pharmacokinetics and the haematological toxicity of the fluorouracil-epirubicin-cyclophosphamide regimen in breast cancer patients. Cancer Chemother Pharmacol, 58: 143-156, 2006.

[12] Latz, J.E., Karlsson, M.O., Rusthoven, J.J., Ghosh, A. and Johnson, R.D. A semimechanisticphysiologic population pharmacokinetic/ pharmacodynamic model for neutropenia following pemetrexed therapy. Cancer Chemother. Pharmacol., 57: 412-426, 2006.

13] Tsukuda. M., Kida, A., Fujii, M., Kono, N., Yoshihara, T., Hasegawa, Y. and Sugita, M. Randomized scheduling feasibility study of S-1 for adjuvant chemotherapy in advanced head and neck cancer. Brit. J. Cancer, 93: 884-889, 2005.

[14] Kimura, Y., Kikkawa, N., Iijima, AS., Kato, T., Naoi, Y., Hayashi, T., Tanigawa, T., Yamamoto, H. and Kurokawa, E. A new regimen for TS-1 therapy designed to minimize adverse reactions by introducing a one-week interval after each two-week dosing session. Jpn. J. Cancer Chemother., 29(8): 1403-1409, 2002.

[15] Chollet, P., Schoffski, P., Weigang, K., Schellens, J.H.M., Cure, H., Pavlidis, N., Grunwald, V., De Boer, R., Wanders, J. and Fumoleau, P. Phase II trial with S-1 in chemotherapy-naïve patients with gastric cancer. A trial performed by the EORTC Early Clinical Studies Group (ECSG). European J. of Cancer, 39: 1264-1270, 2003. 